\title{
Void Shapes Controlled by Using Interruption-Free Epitaxial Lateral Overgrowth of GaN Films on Patterned $\mathrm{SiO}_{2}$ AlN/Sapphire Template
}

\author{
Yu-An Chen, Cheng-Huang Kuo, Li-Chuan Chang, and Ji-Pu Wu \\ Institute of Lighting and Energy Photonics, National Chiao Tung University, Tainan 71150, Taiwan \\ Correspondence should be addressed to Cheng-Huang Kuo; kuoch@mail.nctu.edu.tw
}

Received 20 February 2014; Revised 26 April 2014; Accepted 2 June 2014; Published 18 June 2014

Academic Editor: Nelson Tansu

Copyright (C) 2014 Yu-An Chen et al. This is an open access article distributed under the Creative Commons Attribution License, which permits unrestricted use, distribution, and reproduction in any medium, provided the original work is properly cited.

$\mathrm{GaN}$ epitaxial layers with embedded air voids grown on patterned $\mathrm{SiO}_{2} \mathrm{AlN} /$ sapphire templates were proposed. Using interruptionfree epitaxial lateral overgrowth technology, we realized uninterrupted growth and controlled the shape of embedded air voids. These layers showed improved crystal quality using X-ray diffraction and measurement of etching pits density. Compared with conventional undoped-GaN film, the full width at half-maximum of the GaN (llll $\left.\begin{array}{lll}0 & 0 & 2\end{array}\right)$ and $\left(\begin{array}{lll}1 & 0 & 2\end{array}\right)$ peaks decreased from 485 arcsec to 376 arcsec and from 600 arcsec to 322 arcsec, respectively. Transmission electron microscopy results showed that the coalesced $\mathrm{GaN}$ growth led to bending threading dislocation. We also proposed a growth model based on results of scanning electron microscopy.

\section{Introduction}

III-V compound semiconductors of AlN, GaN, and InN are suitable materials for light-emitting diodes (LEDs) because of their wurtzite crystal structures and direct band gap characteristics [1]. LEDs used for backlighting sources of liquid crystal displays demand solid-state lighting technology [2]. However, large lattice mismatches between substrates and epitaxial layers lead to the formation of threading dislocations (TDs), which decrease the lifetime of diodes and deteriorate the quality of crystals [3]. Epitaxial films with high crystal qualities are necessary for next-generation applications. Hence, reducing the TD density of epitaxial films is a primary challenge.

Recent studies have proposed several useful growth techniques to improve the crystal quality, such as epitaxial lateral overgrowth (ELOG) [4-6], pendeo-epitaxy (PE) [7], maskless PE [8], cantilever epitaxy [9], facet-controlled epitaxial lateral overgrowth (FACELO) $[10,11], \mathrm{SiN}_{x} / \mathrm{GaN}$ buffer layer [12], abbreviated growth mode [13-15], and freestanding GaN substrates [16-18]. Patterned sapphire substrate [19] and embedded air voids method $[20,21]$ have been developed to further enhance the light extraction efficiency (LEE) of light-emitting diodes (LEDs). However, embedded air voids method has been widely used. Several kinds of lateral overgrowth techniques have been reported to create air voids, such as using nanocolumns [22], nanorod [23], PE, or ELOG technique [24]. Ali et al. also showed that void shapes can be controlled using different hexagonally patterned maskless $\mathrm{GaN}$ templates. The TDs near the voids were bent differently with the various hexagonally patterned maskless $\mathrm{GaN}$ templates [25, 26]. Martinez-Criado et al. used the ELOG technique to embed air voids into $\mathrm{GaN}$ substrate and recommended the stress relaxation and crack suppression [16]. Dai et al. reported the higher light escaping probability with the chemical etched embedded rhombus-like air voids in lightemitting structures [27]. In addition, embedded air voids play a key role in freestanding GaN substrate fabrication. Lin et al. employed the GaN films grown on patterned sapphire substrate with large voids on the top region in the chemical lift-off process and found that the embedded voids can accelerate the wet etching process [17]. Bohyama et al. acquired spontaneously separated freestanding GaN substrate by concentrating the compressive stress at the seeds 


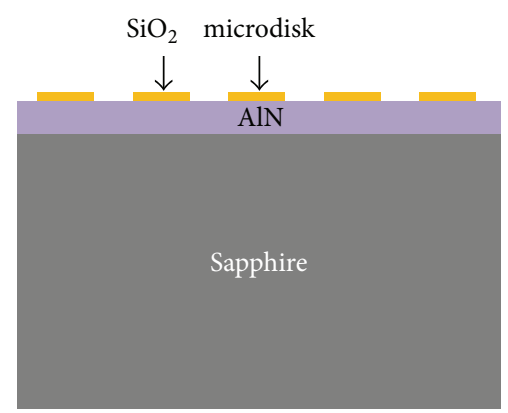

(a)

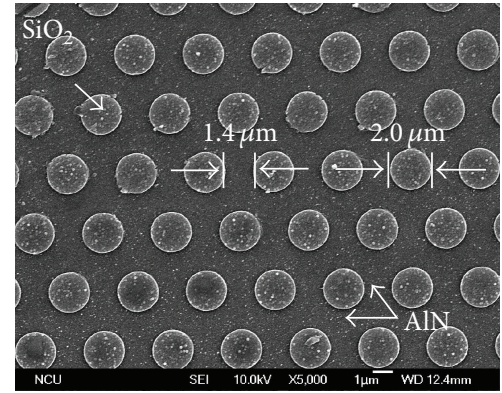

(b)

Figure 1: (a) Cross-sectional structure of patterned $\mathrm{SiO}_{2}$ AlN/sapphire template. (b) Tilted SEM image of patterned $\mathrm{SiO}{ }_{2}$ AlN/sapphire template.

because of the intentional formation of voids [18]. Nevertheless, the above-mentioned techniques inevitably suffer from growth interruption and complicated procedures. In ELOG technique, a $2 \mu \mathrm{m}$-thick GaN epitaxial layer is deposited on the substrate, followed by photolithography with dry etching and photoresist techniques to obtain the templates [4-6]. To satisfy the next-generation application, a high efficiency with a low-cost fabrication method is required. Recently, Lai et al. reported an ex situ AlN buffer layer deposited by sputter, which yielded an interruption-free GaN epitaxy [28]. To further improve the light extraction efficiency, Sheu et al. implanted Ar into a sputtered AlN nucleation layer, and their results showed that the GaN-based epitaxial layer grown on implanted regions has lower growth rates than the implantation-free regions, which eventually form the embedded air voids [29-31].

In this study, we introduced the interruption-free epitaxial lateral overgrowth (IFELOG) technology, a relatively efficient technique developed to simplify template fabrication while keeping the advantages of ELOG. This technology can also control void shapes using a patterned $\mathrm{SiO}_{2} \mathrm{AlN} /$ sapphire template and obtain an uninterrupted growth in metalorganic chemical vapor deposition (MOCVD).

\section{Experimental Procedure}

GaN films used in this study were all prepared by Thomas Swan $\left(3 \times 2^{\prime \prime}\right)$ MOCVD. A $25 \mathrm{~nm}$ thick AlN buffer layer was initially deposited on a c-plane sapphire substrate by sputter. The AlN plates on the separated sputtering guns were used as the sputtering targets for AlN buffer layer deposition. An $80 \mathrm{~nm}$-thick $\mathrm{SiO}_{2}$ film was deposited on the AlN surface by plasma-enhanced chemical vapor deposition. The sample was subsequently patterned by photolithography with photoresist and dry etching processes to form patterned $\mathrm{SiO}_{2}$ microdisks. High-density plasma was used for $\mathrm{SiO}_{2}$ etching. Figure 1(a) shows the specification of the patterned $\mathrm{SiO}_{2} \mathrm{AlN} /$ sapphire template. The pitch and the diameter of the patterned $\mathrm{SiO}_{2}$ microdisk were 3.4 and $2 \mu \mathrm{m}$. Figure 1(b) shows the tilted view of the scanning electron microscope (SEM) image of the patterned $\mathrm{SiO}_{2} \mathrm{AlN} /$ sapphire template.
In this study we controlled void shapes by IFELOG comprising three growth steps, with each step having a specific function. The first step (Step 1) involved the initial formation of GaN seeds exposed on PVD AlN buffer layer. The second step (Step 2) involved the growth performed only against the c-plane growth, and the final step (Step 3) involved the coalescence. Trimethylgallium (TMGa) and ammonia $\left(\mathrm{NH}_{3}\right)$ were, respectively, used as gallium and nitrogen sources during growth. The GaN epitaxial layer grown on a patterned $\mathrm{SiO}_{2}$ AlN/sapphire template with rectangular, triangular, and pillar voids was labeled as sample-R, sample-T, and sample-P, respectively.

At the onset of GaN growth, the growth temperature and chamber pressure in Step 1 were set at $1050^{\circ} \mathrm{C}$ and 400 torr. The growth times of sample- $\mathrm{R}$, sample-T, and sample-P in the same step were 1500,750 , and $1500 \mathrm{~s}$, respectively. The growth temperature and chamber pressure in Step 2 were $1050^{\circ} \mathrm{C}$ and 100 torr. Pulsed growth technique was applied to obtain cplane growth in this step, which is generally used to grow $\mathrm{GaN}$ nanorod arrays [32]. Hence, this technique enhances the cplane growth direction. The respective pulsed growth periods of sample-R, sample-T, and sample-P were 60,60 , and 360 , respectively. The flow rates of TMGa and $\mathrm{NH}_{3}$ were $17 \mathrm{sccm}$ and $3.5 \mathrm{slm}$; the injection times of TMGa and $\mathrm{NH}_{3}$ were 3 and $5 \mathrm{~s}$. Following the pulsed growth, Step 3 was performed with growth temperature and chamber pressure set at $1080^{\circ} \mathrm{C}$ and 400 torr. Conventional undoped-GaN with neither IFELOG technology nor patterned $\mathrm{SiO}_{2} \mathrm{AlN} /$ sapphire template was prepared (i.e., sample-C) for comparative purposes.

The samples were examined by optical microscopy (OM), SEM, X-ray diffraction (XRD), atomic force microscopy (AFM), and transmission electron microscopy (TEM) to discuss the distribution of IFELOG in detail.

\section{Results and Discussion}

Figure 2 shows the cross-sectional SEM images of GaN epitaxial layers with differently shaped air voids utilizing IFELOG on the same template. Figure 2(a) shows the crosssectional SEM images of sample-R with embedded rectangular air voids, each having a width and height of 1.00 and 


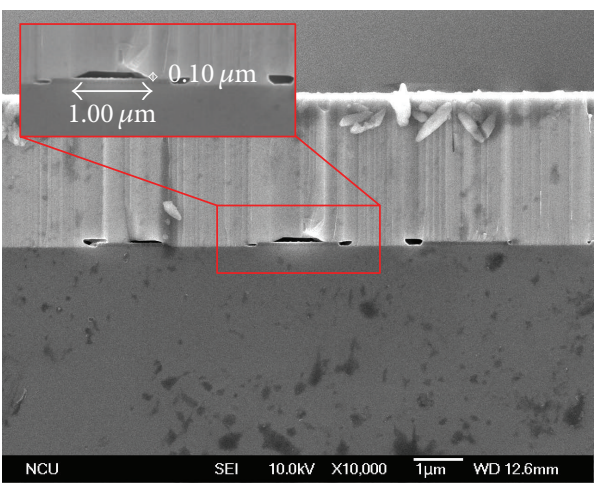

(a)

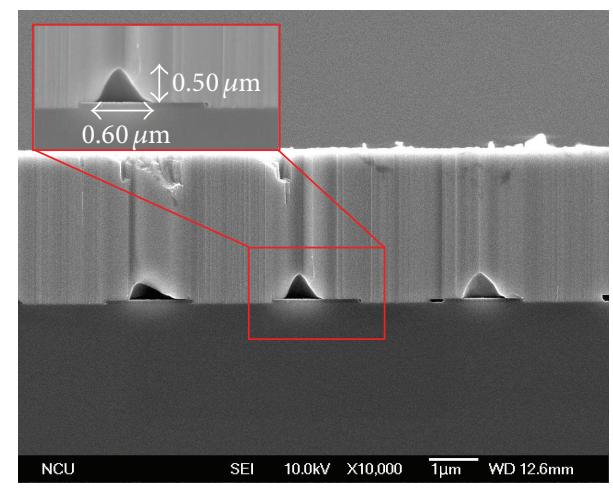

(b)

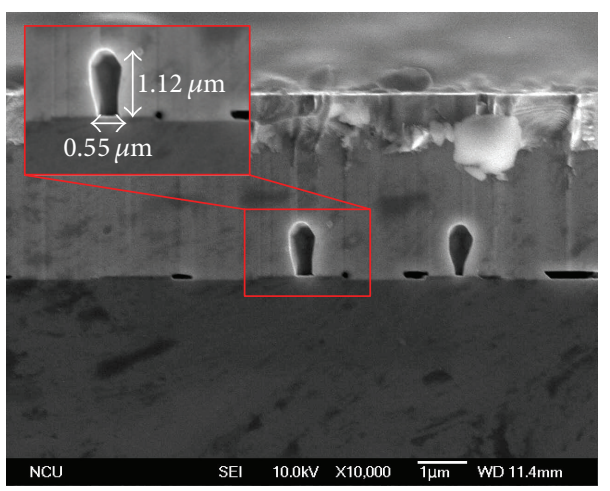

(c)

Figure 2: Cross-sectional SEM images of (a) sample-R, (b) sample-T, and (c) sample-P.

$0.10 \mu \mathrm{m}$. Figure $2(\mathrm{~b})$ shows the cross-sectional SEM images of sample-T with embedded triangular air voids, each having a width and height of 0.60 and $0.50 \mu \mathrm{m}$; the angle of inclination of the voids was $54^{\circ}$. Figure 2 (c) shows the cross-sectional SEM images of sample-P with embedded pillar-shaped air voids, each having a width and height of 0.55 and $1.12 \mu \mathrm{m}$.

Figure 3(a) shows the growth model of the GaN epitaxial layer with various air voids. At the onset of IFELOG, we obtained different distances between $\mathrm{GaN}$ seeds by adjusting the growth time in Step 1 (Figures 3(a-2) and 3(b-2)). We also controlled the void heights by changing the pulsed growth periods in Step 2 (Figures 3(a-3) and 3(c-3)). Figure 4 shows the tilted and cross-sectional SEM images of the GaN epitaxial layers of sample-R after Step-2 growth in the IFELOG technology. The standing GaN seeds confirmed that this technique induced c-plane growth. We also found that the GaN seeds were not able to deposit the entire AlN area during Step-1 growth, which will be discussed in more detail in Figure 7. Considering that the samples had different growth time combinations in between steps, we obtained the various diameters and heights of $\mathrm{GaN}$ seeds. Ali etal. reported that the diameter of hexagonal holes between GaN affects void shape control [25]. In other words, the distances between $\mathrm{GaN}$ seeds define the void shape after coalescence. Figures 3(a-4), 3(b-4), and 3(c-4) show the coalesced growth in Step-3 growth. Narrowing the gap inhibits the source gas molecules from diffusing to the bottom, which eventually forms the
TABLE 1: Full width at half-maximum values of $\omega$ rocking curves measured by XRD.

\begin{tabular}{lccccc}
\hline \multirow{2}{*}{ Sample } & \multicolumn{5}{c}{ FWHM (arcsecs) } \\
& $(002)$ & $(004)$ & $(006)$ & $(102)$ & $(105)$ \\
\hline Sample-C & 485 & 479 & 480 & 600 & 603 \\
Sample-R & 376 & 362 & 355 & 322 & 384 \\
Sample-T & 433 & 454 & 420 & 408 & 459 \\
Sample-P & 416 & 420 & 409 & 360 & 426 \\
\hline
\end{tabular}

embedded air voids that are responsible for the formation of differently shaped air voids (i.e., sample-R, sample-T, and sample-P) [33].

The crystal quality of the GaN epitaxial layer was investigated by XRD and AFM. Heying et al. reported the pure edge TD to be insensitive to the symmetric $\left(\begin{array}{lll}0 & 0 & 1\end{array}\right)$ rocking curves with $l$ nonzero and to distort only the ( $h k l)$ planes with either $h$ or $k$ nonzero. In other words, the decrease in the FWHM values is regarded as a reduction in TDs [34-36]. Table 1 shows the respective values of full width at half maximum (FWHM) of the GaN ( $\left(\begin{array}{lll}0 & 0 & 2\end{array}\right)$ peak, GaN

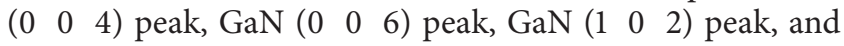
GaN (1 $\left.0 \begin{array}{ll}1 & 0\end{array}\right)$ peak for sample-C, sample-R, sample-T, and sample-P, respectively. All of the XRD results showed an improvement in IFELOG, particularly for sample-R, which 


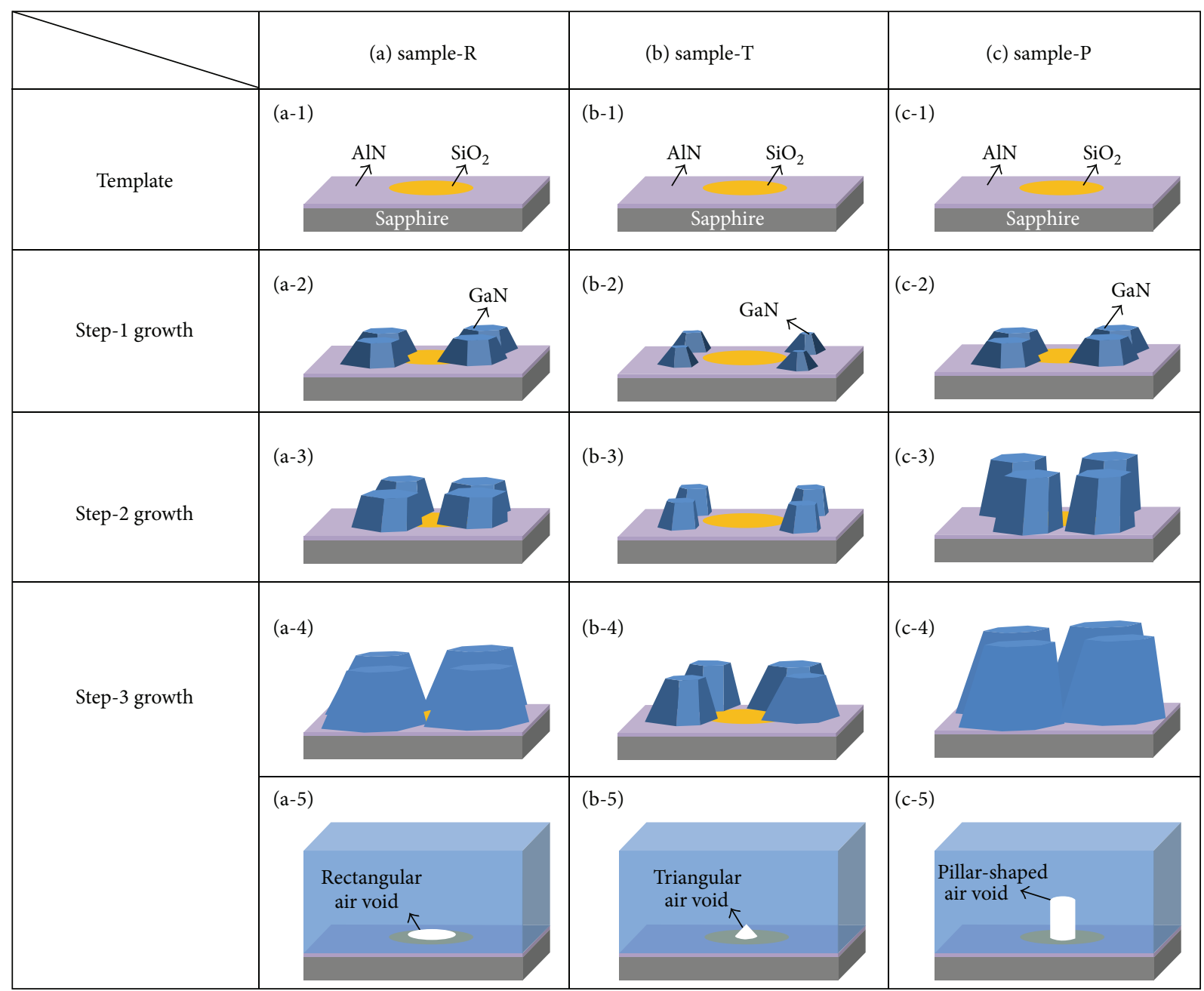

FIGURE 3: The proposed growth model of (a) sample-R, (b) sample-T, and (c) sample-P.

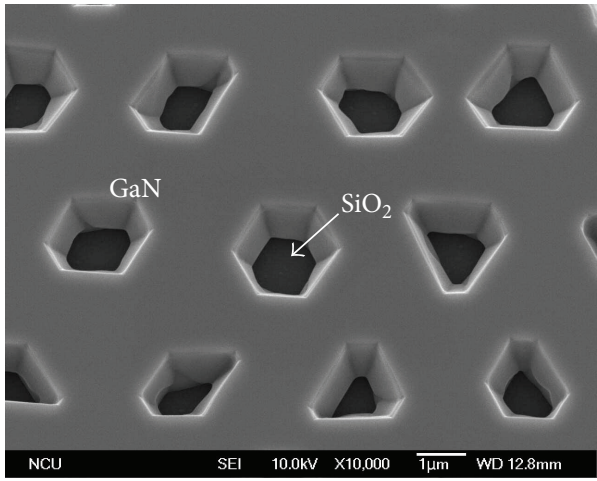

(a)

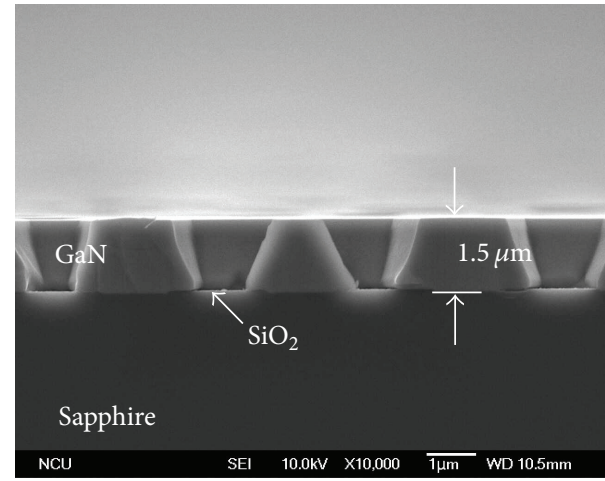

(b)

FIGURE 4: (a) Tilted and (b) cross-sectional SEM images of the sample-R GaN epitaxial layers after Step-1 and Step-2 growth in the IFELOG technology. 


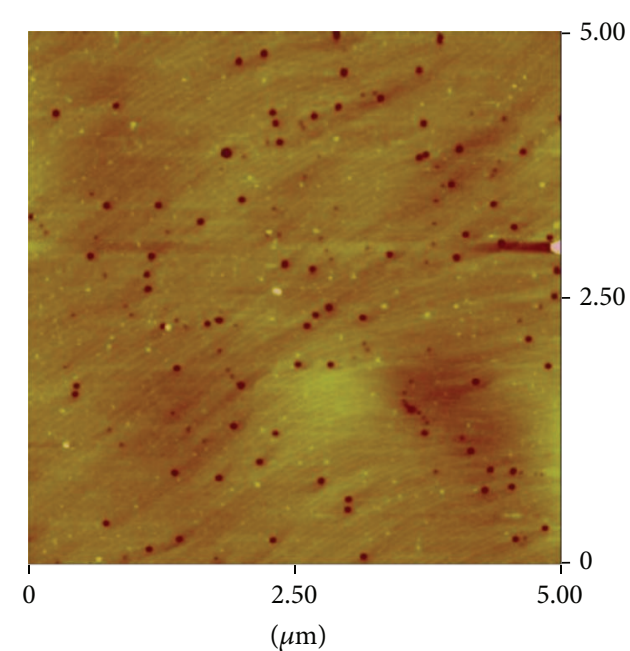

(a)

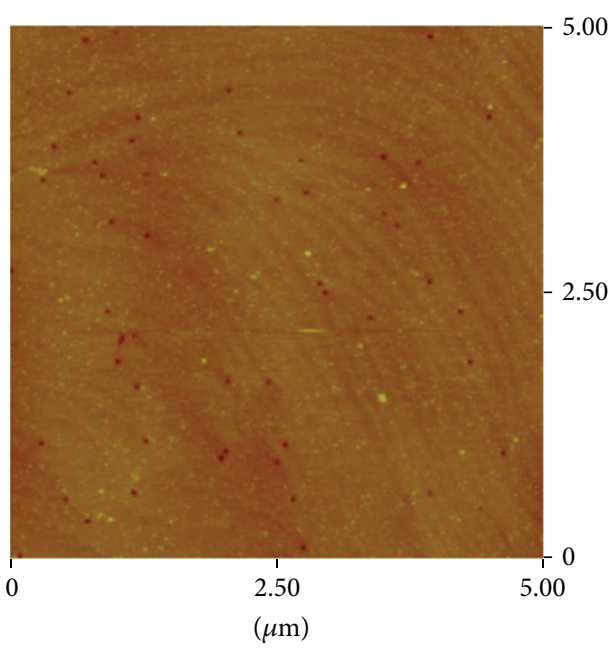

(c)
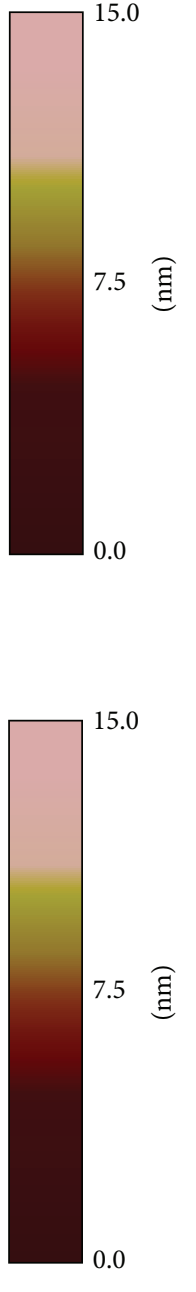

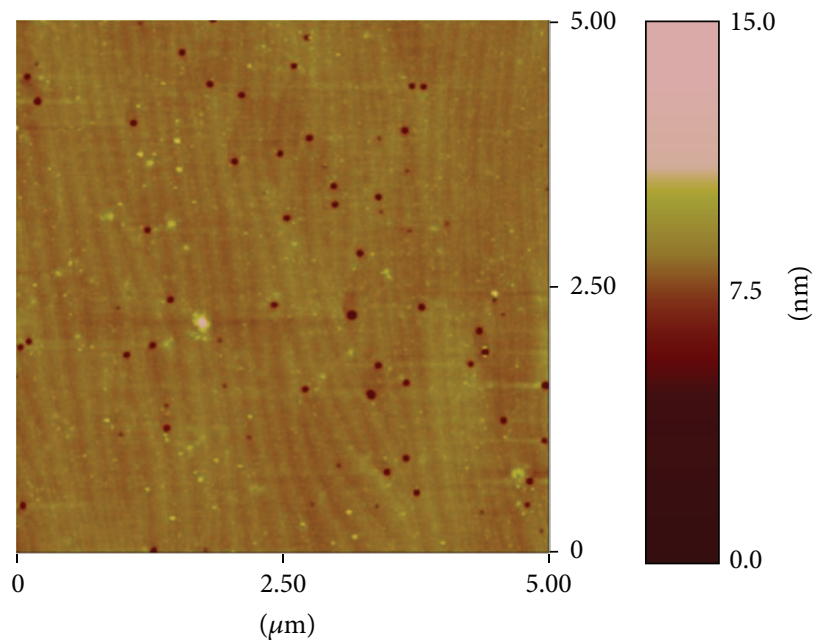

(b)

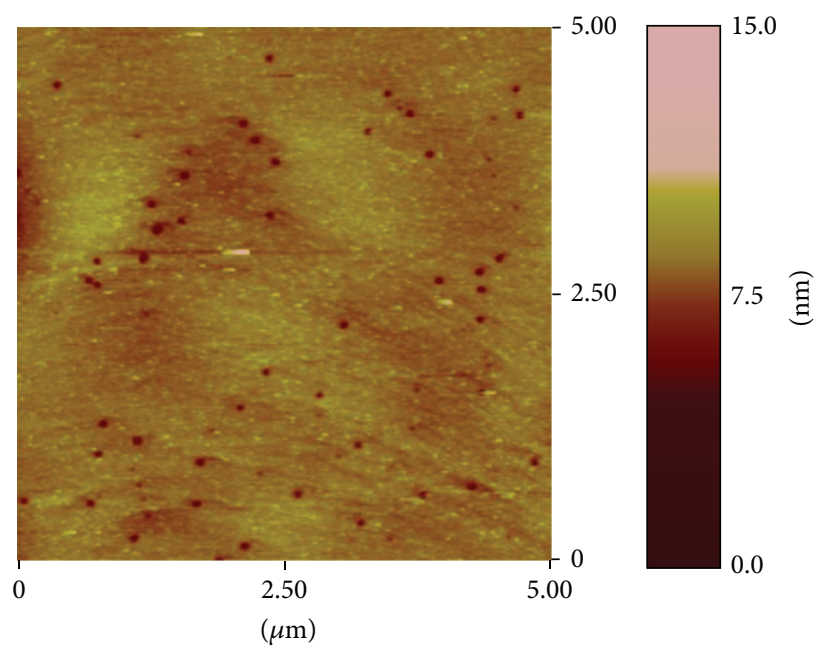

(d)

FIGURE 5: The EPD images over $5 \times 5 \mu \mathrm{m}^{2}$ scanning area of (a) sample-C, (b) sample-R, (c) sample-T, and (d) sample-P.

reduced the GaN (lll $\left.\begin{array}{ll}1 & 2\end{array}\right)$ from 600 arcsec to 322 arcsec. XRD data showed that IFELOG significantly enhanced the crystal quality due to the lateral growth beside the embedded air voids. The bending dislocations were observed in the TEM images.

A wet etching experiment was conducted in $\mathrm{H}_{3} \mathrm{PO}_{4}$ solution at $250^{\circ} \mathrm{C}$ to determine the etching pit densities (EPDs) for $\mathrm{GaN}$ samples, and the samples were then examined using AFM. After wet etching, numerous hexagonal etching pits were observed on the surface. These etching pits were produced by the threading dislocations propagating to the surface of $\mathrm{GaN}$, which originate from the interface between $\mathrm{GaN}$ and substrate. Figure 5 shows the EPD images over a $5 \times 5 \mu \mathrm{m}^{2}$ scanning area of sample-C, sample-R, sample$\mathrm{T}$, and sample-P. The EPDs of sample-C, sample- $\mathrm{R}$, sample$\mathrm{T}$, and sample-P were $5.3 \times 10^{8}, 2.4 \times 10^{8}, 2.7 \times 10^{8}$, and $2.2 \times 10^{8} / \mathrm{cm}^{2}$, respectively. These results indicated that the dislocation densities could be reduced in $\mathrm{GaN}$ epilayer using
IFELOG technology, which also corresponded to the XRD result.

TEM was used to analyze the reduction in the dislocation density. Figure 6 shows the TEM images of the GaN epitaxial layer overgrown on the patterned $\mathrm{SiO}_{2} \mathrm{AlN} /$ sapphire template. The bended TDs were led by the coalesced growth beside the voids [26], which eventually developed into stacking faults (inset of Figure 6(c). Recent studies have reported that TDs can be blocked by $\mathrm{SiO}_{2}$ and stacking faults mentioned above $[37,38]$. Based on the TEM images, the decrease in FWHM of the GaN peak and EPDs was caused by the significant decrease in TDs of the GaN epitaxial layer through IFELOG.

Figures 7(a), 7(b), and 7(c) show the OM images of sample-R, sample-T, and sample-P, respectively. The OM images show that the patterned $\mathrm{SiO}_{2}$ microdisk was surrounded by expanded voids. Among all the samples, sample-R and sample- $T$ had the most and least numbers of expanded 


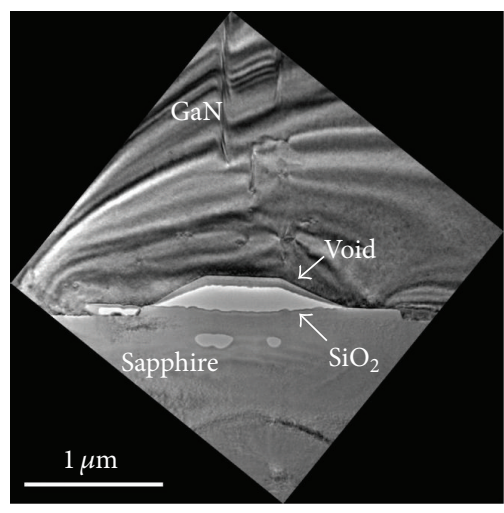

(a)

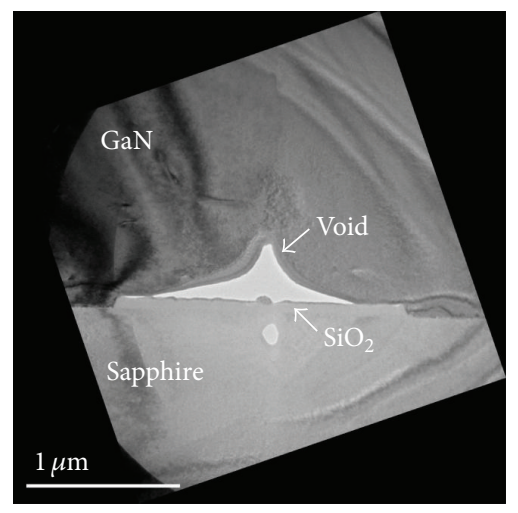

(b)

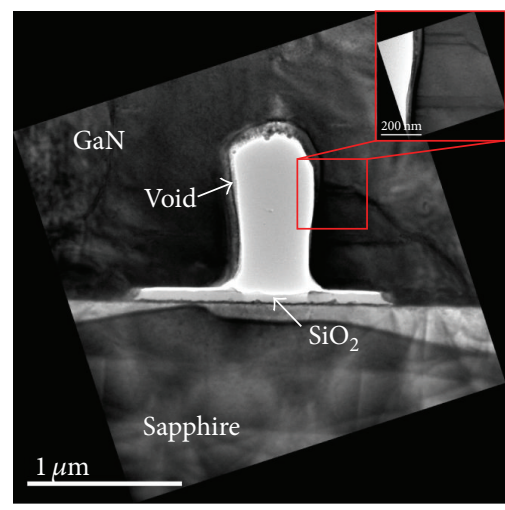

(c)

Figure 6: The TEM images of (a) sample-R, (b) sample-T, and (c) sample-P.

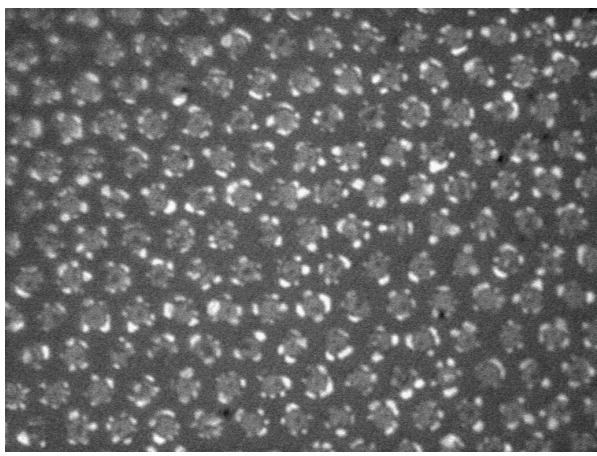

(a)

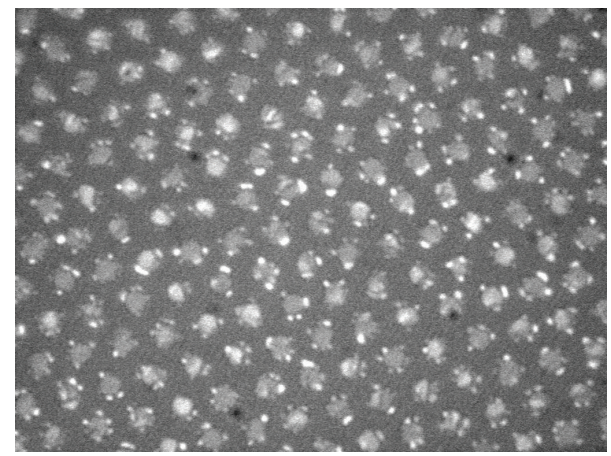

(b)

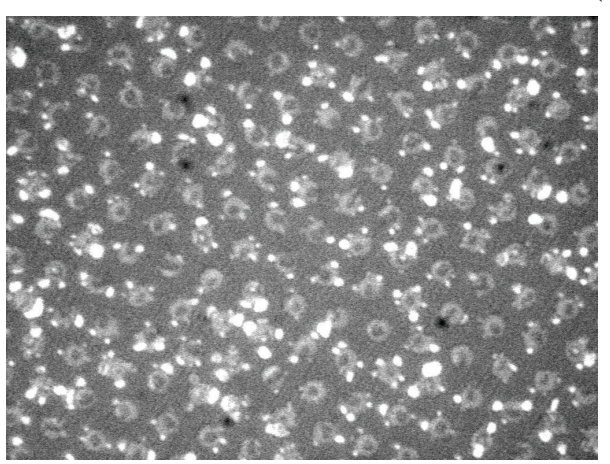

(c)

FIgURE 7: The OM images of (a) sample-R, (b) sample-T, and (c) sample-P. 


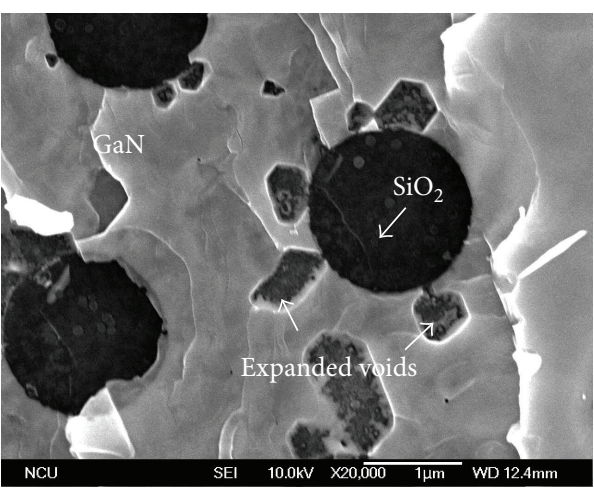

(a)

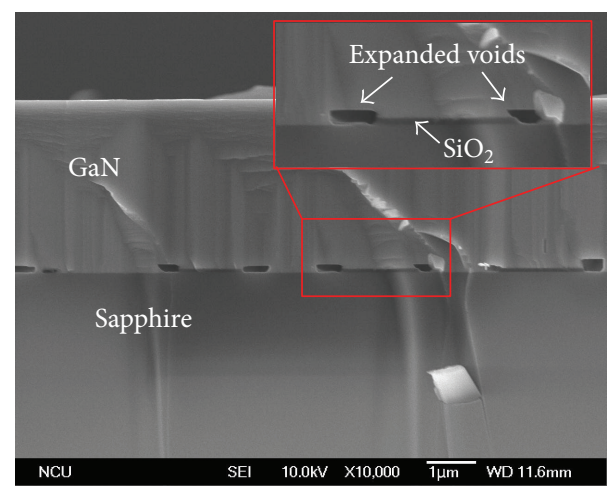

(b)

FIGURE 8: (a) The SEM image of sample-R lifted off by diamond cutter. (b) The cross-sectional SEM image of sample-R.

voids. Lift-off template surface and cross-section SEM measurements were performed to further clarify the cause of the expanded voids that surrounded the patterned $\mathrm{SiO}_{2}$ microdisk. Sample-R was lifted off by a diamond cutter and it was found that the expanded voids surrounded the patterned $\mathrm{SiO}_{2}$ microdisk (Figure 8(a)). Figure 8(b) shows the cross-sectional SEM images prepared to clearly observe the interface between $\mathrm{SiO}_{2}$ and the $\mathrm{GaN}$ epitaxial layer. The formation of expanded voids was caused by the discontinuous island-type growth characteristic of the GaN seed layer in the beginning. In other words, GaN seeds were not able to deposit the entire AlN area during Step-1 growth, which resulted in residual vacancies beside the patterned $\mathrm{SiO}_{2}$ microdisk. These vacancies were converted into expanded voids, which had the similar function with the voids generated from the nanorod template [23]. Such behavior can increase the lateral growth and lower the TDs. These expanded voids were suggested to have positive effects on the quality of the GaN epitaxial layer because of the enhancement in XRD data and EPD result of sample-R, which had the most number of expanded voids.

\section{Conclusion}

We successfully demonstrated an interruption-free epitaxial lateral overgrowth technology by combining sputter AlN buffer layer and pulsed growth method. By adjusting Step1 growth time and the periods of Step- 2 growth, we easily controlled the void shape by the same template. The growth model was proposed to explain the formation of differently shaped air voids based on the SEM results. AFM images show that the epitaxial layer grown by IFELOG technology has lower etching pit densities, thereby increasing the volume of defect-free regions and bending TDs. XRD data suggests

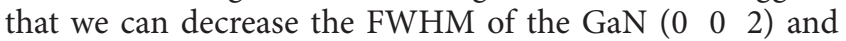
$\left(\begin{array}{lll}1 & 0 & 2\end{array}\right)$ peaks from 485 arcsec to 376 arcsec and from 600 arcsec to 322 arcsec in sample-R. IFELOG technology not only simplified the fabrication of templates, but also greatly enhanced the quality of the GaN epitaxial layer and yielded an uninterrupted growth.

\section{Conflict of Interests}

The authors declare that there is no conflict of interests regarding the publication of this paper.

\section{Acknowledgment}

The authors would like to acknowledge the financial support from National Science Council of Taiwan for their research Grants of NSC 101-2221-E-009-028-MY3 and NSC-3113-P009-007-CC2.

\section{References}

[1] T. Mukai, M. Yamada, and S. Nakamura, "Characteristics of InGaN-based UV/blue/green/amber/red light-emitting diodes," Japanese Journal of Applied Physics, Part 1: Regular Papers and Short Notes and Review Papers, vol. 38, no. 7, pp. 3976-3981, 1999.

[2] Y. Narukawa, I. Niki, K. Izuno, M. Yamada, Y. Murazaki, and T. Mukai, "Phosphor-conversion white light emitting diode using InGaN near-ultraviolet chip," Japanese Journal of Applied Physics, Part 2: Letters, vol. 41, no. 4, pp. L371-L373, 2002.

[3] Y. P. Hsu, S. J. Chang, Y. K. Su et al., "Lateral epitaxial patterned sapphire InGaN/GaN MQW LEDs," Journal of Crystal Growth, vol. 261, no. 4, pp. 466-470, 2004.

[4] D. Kapolnek, S. Keller, R. Vetury et al., "Anisotropic epitaxial lateral growth in GaN selective area epitaxy," Applied Physics Letters, vol. 71, no. 9, pp. 1204-1206, 1997.

[5] T. S. Zheleva, O.-H. Nam, M. D. Bremser, and R. F. Davis, "Dislocation density reduction via lateral epitaxy in selectively grown GaN structures," Applied Physics Letters, vol. 71, no. 17, pp. 2472-2474, 1997.

[6] M. Hansen, P. Fini, M. Craven, B. Heying, J. S. Speck, and S. P. DenBaars, "Morphological and optical properties of InGaN laser diodes on laterally overgrown GaN," Journal of Crystal Growth, vol. 234, no. 4, pp. 623-630, 2002.

[7] I.-H. Kim, C. Sone, O.-H. Nam, Y.-J. Park, and T. Kim, “Crystal tilting in GaN grown by pendoepitaxy method on sapphire substrate," Applied Physics Letters, vol. 75, no. 26, pp. 4109-4111, 1999. 
[8] A. M. Roskowski, E. A. Preble, S. Einfeldt, P. M. Miraglia, and R. F. Davis, "Investigations regarding the maskless Pendeoepitaxial growth of GaN films prior to coalescence," IEEE Journal of Quantum Electronics, vol. 38, no. 8, pp. 1006-1016, 2002.

[9] C. I. H. Ashby, C. C. Mitchell, J. Han et al., "Low-dislocationdensity GaN from a single growth on a textured substrate," Applied Physics Letters, vol. 77, no. 20, pp. 3233-3235, 2000.

[10] H. Miyake, R. Takeuchi, K. Hiramatsu et al., "High quality GaN grown by facet-controlled ELO, (FACELO) technique," Physica Status Solidi (A), vol. 194, pp. 545-549, 2002.

[11] K. Hiramatsu, K. Nishiyama, M. Onishi et al., "Fabrication and characterization of low defect density GaN using facetcontrolled epitaxial lateral overgrowth (FACELO)," Journal of Crystal Growth, vol. 221, no. 1-4, pp. 316-326, 2000.

[12] Y.-B. Lee, T. Wang, Y.-H. Liu et al., "High-performance 348 nm AlGaN/GaN-based ultraviolet-light-emitting diode with a SiN buffer layer," Japanese Journal of Applied Physics, Part 1: Regular Papers and Short Notes and Review Papers, vol. 41, no. 7, pp. 4450-4453, 2002.

[13] Y.-K. Ee, J. M. Biser, W. Cao, H. M. Chan, R. P. Vinci, and N. Tansu, "Metalorganic vapor phase epitaxy of III-nitride lightemitting diodes on nanopatterned AGOG sapphire substrate by abbreviated growth mode," IEEE Journal on Selected Topics in Quantum Electronics, vol. 15, no. 4, pp. 1066-1072, 2009.

[14] Y.-K. Ee, X.-H. Li, J. Biser et al., "Abbreviated MOVPE nucleation of III-nitride light-emitting diodes on nano-patterned sapphire," Journal of Crystal Growth, vol. 312, no. 8, pp. 1311-1315, 2010.

[15] W. Cao, J. M. Biser, Y.-K. Ee et al., "Dislocation structure of GaN films grown on planar and nano-patterned sapphire," Journal of Applied Physics, vol. 110, no. 5, Article ID 053505, 2011.

[16] G. Martinez-Criado, M. Kuball, M. Benyoucef et al., "Freestanding GaN grown on epitaxial lateral overgrown $\mathrm{GaN}$ substrates," Journal of Crystal Growth, vol. 255, no. 3-4, pp. 277281, 2003.

[17] C.-F. Lin, J.-J. Dai, M.-S. Lin et al., "An AlN sacrificial buffer layer inserted into the GaN/patterned sapphire substrate for a chemical lift-off process," Applied Physics Express, vol. 3, no. 3, Article ID 031001, 2010.

[18] S. Bohyama, H. Miyake, K. Hiramatsu, Y. Tsuchida, and T. Maeda, "Freestanding GaN substrate by advanced facet-controlled epitaxial lateral overgrowth technique with masking side facets," Japanese Journal of Applied Physics, Part 2: Letters, vol. 44, no. 1-7, pp. L24-L26, 2005.

[19] W.-K. Wang, D.-S. Wuu, W.-C. Shih et al., "Near-ultraviolet InGaN/GaN light-emitting diodes grown on patterned sapphire substrates," Japanese Journal of Applied Physics, Part 1: Regular Papers and Short Notes and Review Papers, vol. 44, no. 4, pp. 2512-2515, 2005.

[20] W.-C. Lai, Y.-Y. Yang, L.-C. Peng, S.-W. Yang, Y.-R. Lin, and J.K. Sheu, "GaN-based light emitting diodes with embedded $\mathrm{SiO}_{2}$ pillars and air gap array structures," Applied Physics Letters, vol. 97, no. 8, Article ID 081103, 2010.

[21] M. Ali, O. Svensk, L. Riuttanen et al., "Enhancement of near-UV GaN LED light extraction efficiency by GaN/sapphire template patterning," Semiconductor Science and Technology, vol. 27, no. 8, Article ID 082002, 2012.

[22] S. D. Hersee, X. Y. Sun, X. Wang, M. N. Fairchild, J. Liang, and J. Xu, "Nanoheteroepitaxial growth of GaN on Si nanopillar arrays," Journal of Applied Physics, vol. 97, no. 12, Article ID 124308, 2005.
[23] C. H. Kuo, Y. A. Chen, J. P. Wu, and L. C. Chang, "Efficiency improvement of near-ultraviolet nitride-based light-emittingdiode prepared on GaN nano-rod arrays by metalorganic chemical vapor deposition," IEEE Journal of Quantum Electronics, vol. 50, no. 3, article 129, 2014.

[24] Z. Liliental-Weber, X. Ni, and H. Morkoc, "Structural perfection of laterally overgrown GaN layers grown in polar- and nonpolar directions," Journal of Materials Science: Materials in Electronics, vol. 19, no. 8-9, pp. 815-820, 2008.

[25] M. Ali, A. E. Romanov, S. Suihkonen et al., "Void shape control in GaN re-grown on hexagonally patterned mask-less GaN," Journal of Crystal Growth, vol. 315, no. 1, pp. 188-191, 2011.

[26] M. Ali, A. E. Romanov, S. Suihkonen et al., "Analysis of threading dislocations in void shape controlled GaN re-grown on hexagonally patterned mask-less GaN," Journal of Crystal Growth, vol. 344, no. 1, pp. 59-64, 2012.

[27] J.-J. Dai, C.-F. Lin, G.-M. Wang, and M.-S. Lin, "Enhanced the light extraction efficiency of an InGaN light emitting diodes with an embedded rhombus-like air-void structure," Applied Physics Express, vol. 3, no. 7, Article ID 071002, 2010.

[28] C.-H. Yen, W.-C. Lai, Y.-Y. Yang et al., "GaN-based lightemitting diode with sputtered AlN nucleation layer," IEEE Photonics Technology Letters, vol. 24, no. 4, pp. 294-296, 2012.

[29] Y. H. Yeh, J. K. Sheu et al., "InGaN flip-chip light emitting diodes with embedded air voids as light-scattering layer," IEEE Electron Device Letters, vol. 34, no. 12, pp. 1542-1544, 2013.

[30] J.-K. Sheu, Y.-H. Yeh, S.-J. Tu, M.-L. Lee, P. C. Chen, and W.C. Lai, "Improved output power of GaN-based blue LEDs by forming air voids on Ar-implanted sapphire substrate," Journal of Lightwave Technology, vol. 31, no. 8, Article ID 6464504, pp. 1318-1322, 2013.

[31] J.-K. Sheu, S.-J. Tu, Y.-H. Yeh, M.-L. Lee, and W.-C. Lai, "Gallium nitride-based light-emitting diodes with embedded air voids grown on Ar-implanted AlN/sapphire substrate," Applied Physics Letters, vol. 101, no. 15, Article ID 151103, 2012.

[32] S. D. Hersee, X. Sun, and X. Wang, "The controlled growth of GaN nanowires," Nano Letters, vol. 6, no. 8, pp. 1808-1811, 2006.

[33] H. J. Oh, S. W. Rhee, and I. S. Kang, "Simulation of CVD process by boundary integral technique," Journal of the Electrochemical Society, vol. 139, no. 6, pp. 1714-1720, 1992.

[34] H. Heinke, V. Kirchner, S. Einfeldt, and D. Hommel, "X-ray diffraction analysis of the defect structure in epitaxial GaN," Applied Physics Letters, vol. 77, no. 14, pp. 2145-2147, 2000.

[35] Y. Iyechika, M. Shimizu, T. Maeda, H. Miyake, and K. Hiramatsu, "X-ray analysis of twist and tilt of GaN prepared by facetcontrolled epitaxial lateral overgrowth (FACELO)," Japanese Journal of Applied Physics, Part 2: Letters, vol. 42, no. 7, pp. L732L734, 2003.

[36] B. Heying, X. H. Wu, S. Keller et al., "Role of threading dislocation structure on the $\mathrm{x}$-ray diffraction peak widths in epitaxial GaN films," Applied Physics Letters, vol. 68, article 643, 1995.

[37] C. H. Chiu, H. H. Yen, C. L. Chao et al., "Nanoscale epitaxial lateral overgrowth of $\mathrm{GaN}$-based light-emitting diodes on a $\mathrm{SiO}_{2}$ nanorod-array patterned sapphire template," Applied Physics Letters, vol. 93, no. 8, Article ID 081108, 2008.

[38] D. S. Wuu, W. K. Wang, K. S. Wen et al., "Fabrication of pyramidal patterned sapphire substrates for high-efficiency InGaNbased light emitting diodes," Journal of the Electrochemical Society, vol. 153, no. 8, Article ID 063608JES, pp. G765-G770, 2006. 

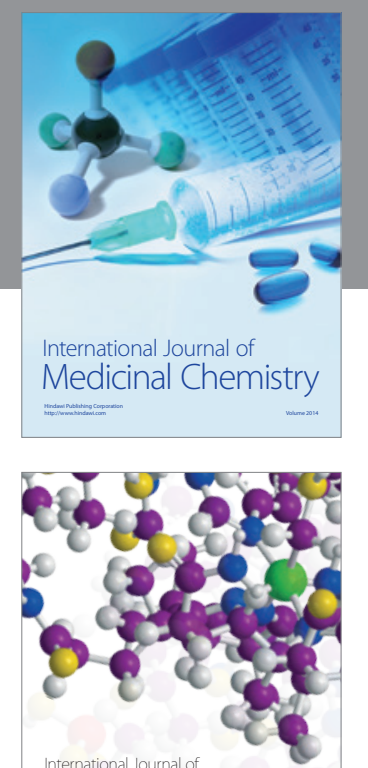

\section{Carbohydrate} Chemistry

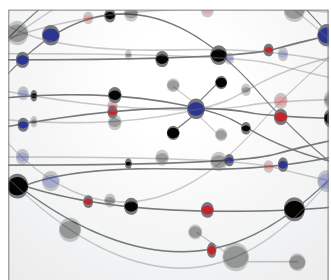

The Scientific World Journal
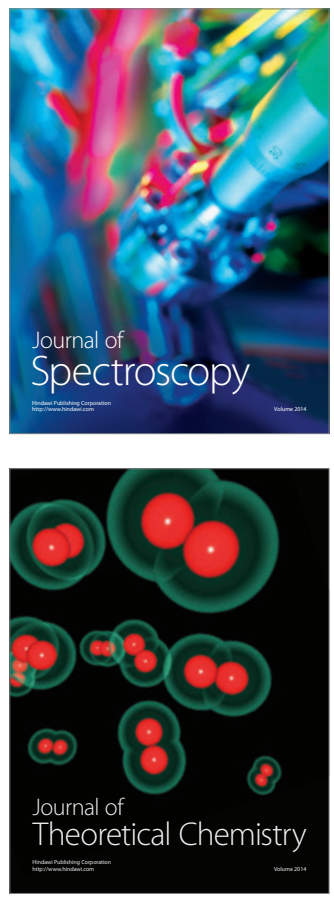
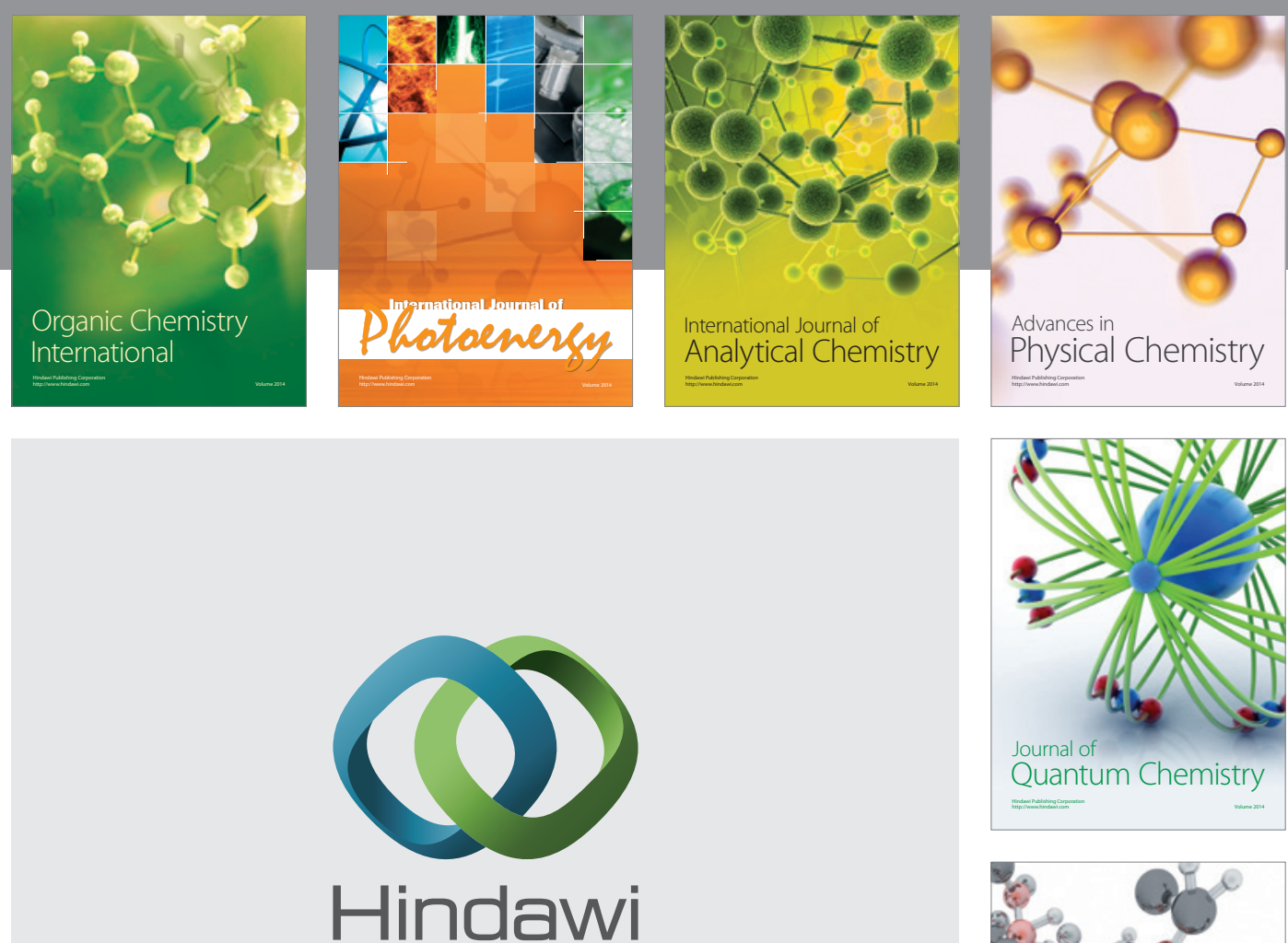

Submit your manuscripts at

http://www.hindawi.com

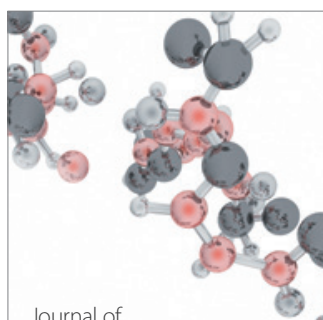

Analytical Methods

in Chemistry

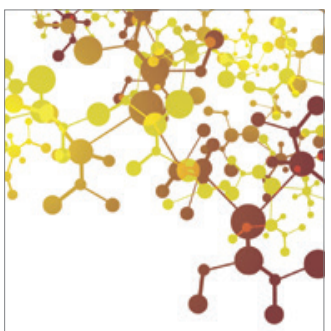

Journal of

Applied Chemistry

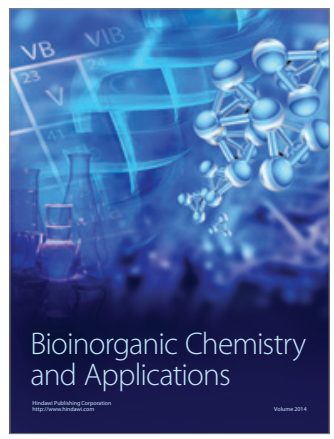

Inorganic Chemistry
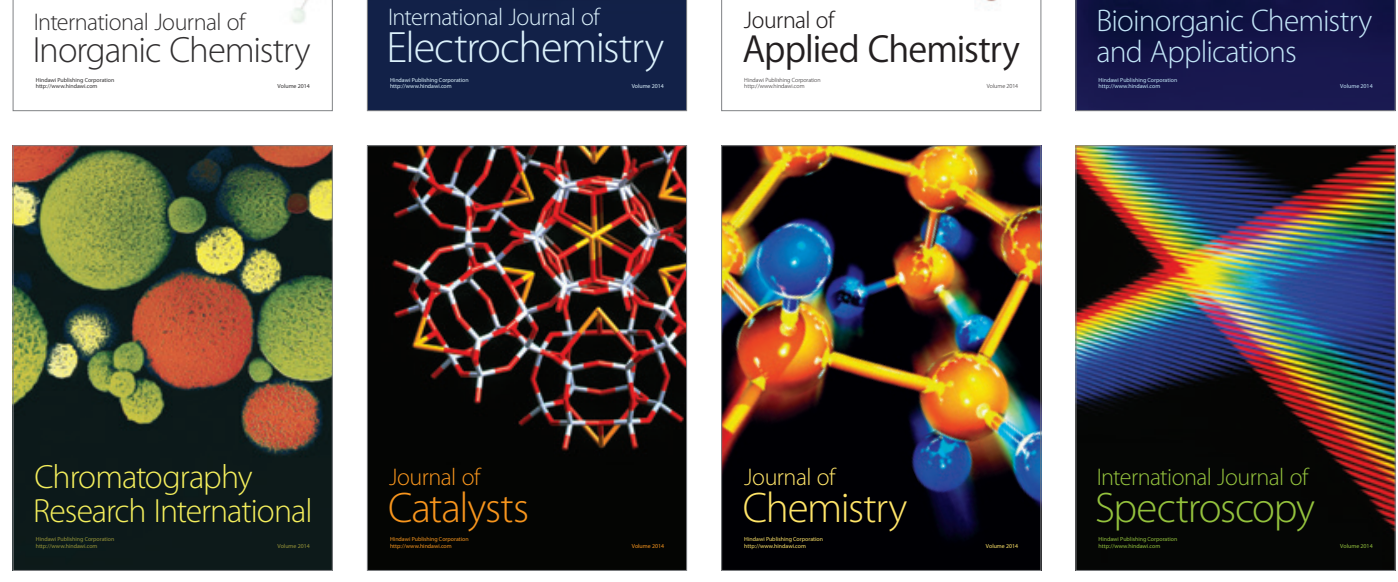